\title{
Innovación Abierta y Eco-Innovación: Capacidades dinámicas para mejorar el desempeño de las PYMEs
}
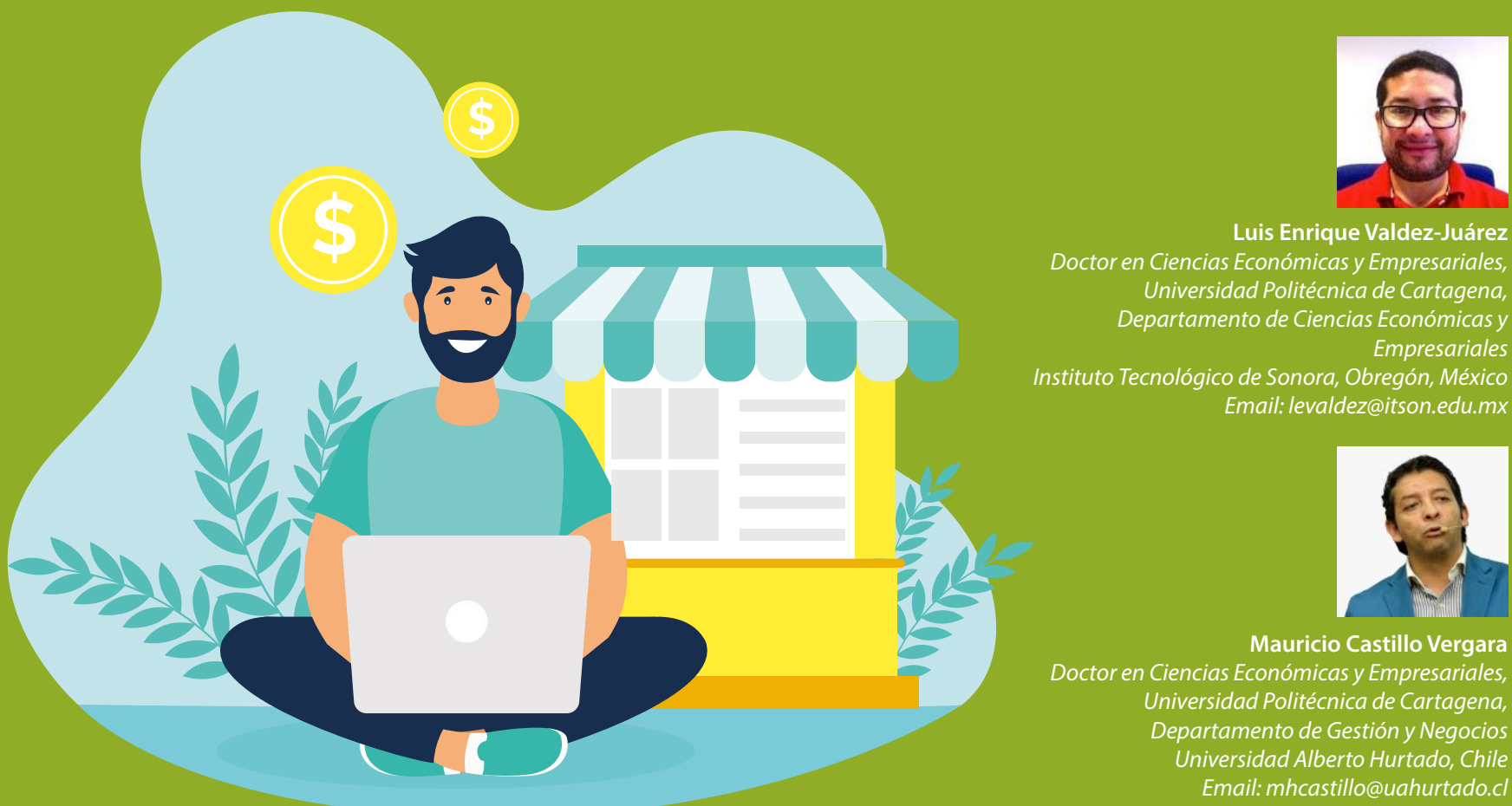

Que las pequeñas y medianas empresas (PYMEs) puedan ser competitivas no es un desafío sencillo, hay una serie de limitaciones que deben sobrellevar. Por eso, estudiamos un conjunto de 684 PYMEs de México utilizando un modelo de ecuaciones estructurales, para determinar qué efectos tiene en el desempeño corporativo el desarrollar capacidades de innovación abierta y eco-innovación.

\section{Introducción}

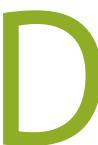
urante mucho tiempo, las empresas han estado trabajando para lograr desarrollar nuevos procesos o productos para incrementar sus niveles de competitividad (Teece, 2010). Sin embargo, los avances tecnológicos, los efectos y cambios de la economía y el comportamiento del mercado han invitado a que muchas organizaciones tengan que volver a pensar sus modelos de negocio (Geissdoerfer et al., 2018). En este camino, los directivos de empresas se han centrado en enfoques cerrados para el desarrollo de actividades innovadoras.

Sin embargo, derivado de la demanda de mercados altamente competitivos, la innovación abierta (open innovation) surge como una estrategia que rompe con paradigmas de innovación cerrados (Chesbrough, 2006). La innovación abierta se refiere principalmente a la apertura del proceso de innovación al conocimiento del exterior de la organización innovadora. La innovación abierta es un paradigma que asume que las empresas pueden y deben utilizar tanto ideas externas como internas, los límites entre una empresa y su ecosistema se volverán cada vez más invisibles y las innovaciones podrán transferirse fácilmente hacia adentro y hacia afuera. Por tanto, la innovación abierta puede conducir a modelos empresariales innovadores (Hans-Dieter Zimmermann \& Pucihar, 2015).

En el contexto de las pequeñas y medianas empresas (PYMEs) de países de economías emergentes, la innovación abierta es una forma novedosa para incorporar capacidades externas a una organización en el desarrollo de innovación. Con esto, es posible eliminar los límites internos e involucrar al resto de stakeholders (gerentes, empleados, clientes, proveedores, sociedad civil, usuarios de la innovación), desarrollando un comportamiento innovador y cambio hacía una cultura basada en la creatividad y la innovación (Valdez-Juárez \& Castillo-Vergara, 2021).

A pesar de estos nuevos paradigmas, estas capacidades ya no son suficientes. Con la irrupción de nuevos requerimientos y desafíos, siendo el deterioro medio ambiental uno de los más urgentes, ha despertado en las empresas y consumidores un mayor interés en el desarrollo de innovaciones sostenibles que permitan una producción sostenible. Este concepto denominado eco-innovación surge como resultado de integrar la filosofía de la sostenibilidad, es un tipo de innovación que busca reducir riesgos, contaminación y consumo energético en comparación con otros alternativas (Leitão et al., 2020). 


\section{"Con la irrupción de nuevos requerimientos y desafíos, siendo el deterioro medio ambiental uno de los más urgentes, ha despertado en las empresas y consumidores un mayor interés en el desarrollo de
innovaciones sostenibles que permitan una producción sostenible"}

El hecho que las sociedades están buscando ser cada vez más responsables en términos de patrones productivos, consumo y comportamiento de compra, invita a las empresas a adoptar estas capacidades para el desarrollo de sus modelos de negocios. La eco-innovación adquirirá una gran relevancia para crear valor no solo económico sino incluyendo también aspectos sociales y ambientales.

La incorporación de estas capacidades no es directa, en particular en las PYMEs en contexto de economías emergentes, dado que tienes capacidades limitadas, personal poco calificado, escasez de recursos y bajo acceso a tecnología. Por lo cual estudiamos cómo las capacidades para desarrollar innovación abierta y eco-innovación pueden ser estimuladas con la incorporación de capacidades tecnológicas y cuantificamos como impactan en el desempeño de las PYMEs. El estudio se realizó en pequeñas y medianas empresas de México, que es un caso muy interesante, dado que ha tenido tasas de crecimiento del PIB favorables desde la década de 1990 (World Bank, 2020), pero su desempeño relativo en término de innovación se ha mantenido por debajo de las expectativas dado el tamaño de su economía. Probamos el modelo teórico de la Figura 1, que incluye capacidades tecnológicas, innovación abierta y eco-innovación con 684 PYMEs.

\section{Datos}

La investigación se llevó a cabo considerando una muestra de la población de Pymes ubicadas en la región noroeste de México. La selección de la muestra es del tipo estratificado y se seleccionaron las Pymes del sector comercio dado que representan una participación económica crucial de México. El tamaño de la empresa se considera según aquellas que tienen entre 10 y 200 empleados (el $85 \%$ son pequeñas empresas de 10 a 50 empleados y el $15 \%$ son empresas medianas de 51 a 200 empleados). La información de las empresas se obtiene del Directorio Nacional de Unidades Económicas (DENUE). La muestra final de 684 Pymes es representativa con un error de $3 \%$ y nivel de confiabilidad de $95 \%$. El trabajo de campo se realizó durante los meses de marzo a noviembre de 2019, mediante el uso de un cuestionario en forma de entrevista personal con el propietario y / o gerente de cada em- presa participante. Del total de empresas, la empresa más antigua tiene 67 años en funcionamiento y la más joven tiene 1 año de actividad en el sector. Un 59,5\% de las empresas es dirigida por hombres y $40,5 \%$ por mujeres.

\section{Resultados}

Los resultados muestran que la capacidad tecnológica de las PYMEs tiene un impacto más significativo en las actividades de innovación abierta, ya que les ayuda a recopilar información, transferirla dentro de la empresa y mejorar procesos y productos. Por otro lado, las capacidades tecnológicas tienen un impacto similar en las actividades de eco-innovación, entonces un factor determinante para su adopción es el impulso al uso de la tecnología, por qué desarrollar innovaciones más limpias
Referencias Harvard Business School Press. Sciences, Organizational, Septembre 9-11, 449-458. https://doi.org/https://doi.org/10.3390/joitmc6040145 https://doi.org/10.1016/j.lrp.2009.07.003 Market, and Complexity, $7(1), 8$. mexico?view=chart es más complejo y requiere una capacidad tecnológica más destacada. En el modelo, la capacidad tecnológica no tiene un impacto significativo en los resultados de desempeño corporativo.

Los hallazgos relacionados con el impacto de la innovación abierta y la eco-innovación en el desempeño corporativo indican que las actividades de innovación abierta llevadas a cabo por las PYMEs tienen un efecto moderado, pero significativo en los resultados del desempeño corporativo. Las empresas pueden crear valor para sus consumidores mediante la innovación abierta, pero para lograrlo, se deben desarrollar otros factores como la creatividad, la capacidad de aprender, la tolerancia al fracaso y la colaboración.

Con un impacto similar y un efecto significativo, la eco-innovación juega un papel fundamental en los resultados de desempeño corporativo de las PYMEs; sin embargo, estas nuevas prácticas para este tipo de empresas son novedosas y requieren mayor madurez, compromiso y prácticas empresariales organizacionales sostenibles para lograr mejores resultados.

Figura 1. Modelo Teórico Propuesto del impacto de capacidades en el desempeño de las PYMEs

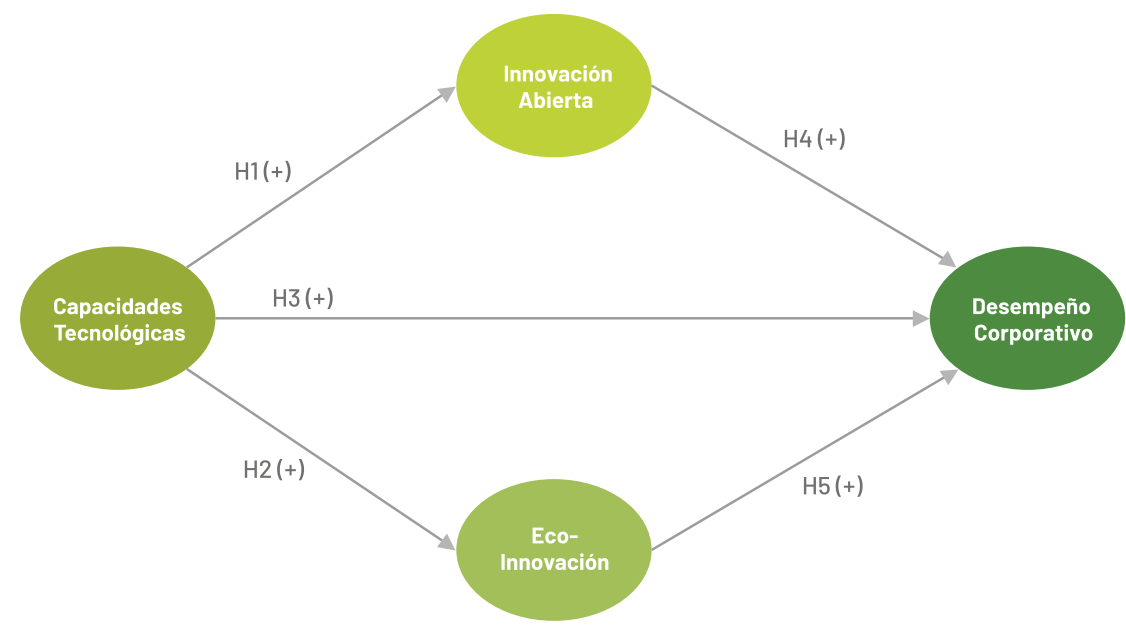

-Chesbrough, H. W. (2006). Open Innovation: The New Imperative for Creating and Profiting from Technology.

- Geissdoerfer, M., Vladimirova, D., \& Evans, S. (2018). Sustainable business model innovation: A review. In Journal of Cleaner Production (Vol. 198, pp. 401-416). Elsevier Ltd. https://doi.org/10.1016/j.jclepro.2018.06.240

- Hans-Dieter Zimmermann, \& Pucihar, A. (2015). OPEN INNOVATION, OPEN DATA AND NEW BUSINESS MODELS.

- Leitão, J., Pereira, D., \& de Brito, S. (2020). Inbound and Outbound Practices of Open Innovation and Eco-Innovation: Contrasting Bioeconomy and Non-Bioeconomy Firms. J. Open Innov. Technol. Mark. Complex, 6(4), 145.

- Teece, D. J. (2010). Business models, business strategy and innovation. Long Range Planning, 43(2-3), 172-194.

-Valdez-Juárez, E. L., \& Castillo-Vergara, M. (2021). Technological Capabilities, Open Innovation, and Eco-Innovation : Dynamic Capabilities to Increase Corporate Performance of SMEs. Journal of Open Innovation: Technology,

-World Bank. (2020). World Development Indicators Database. Databank. https://datos.bancomundial.org/pais/ 